\title{
Transient branch retinal artery occlusion in a 15 -year-old girl and review of the literature
}

\author{
Alexandr Stepanov, Libor Hejsek, Nada Jiraskova, Alena Feuermannova, Eva Rencova, Pavel Rozsival
}

\begin{abstract}
Background. Retinal artery occlusion is an extremely rare diagnosis in the pediatric population and the etiology with risk factors of retinal artery occlusion are poorly understood in younger individuals.

Methods and Results. This case report a rare case of transient branch retinal artery occlusion (BRAO) in a healthy young girl. A fifteen-year-old girl presented with painless decreased vision in her right eye. Fundus examination revealed in the right eye intensive edema of the part of superior half of the retina, but the vessels were absolutely normal. She was hospitalized for diagnosis and treatment. A thorough workup was performed to determine any etiologic factor. Her physical examination was normal, but from anamnesis has been found that patient began use oral contraception at the time of occurrence eye problems. The condition is evaluated as transient branch retinal artery occlusion by the course of the disease. The paper includes the review of the literature also.

Conclusion. Though retinal arterial obstruction is rare in the pediatric population, this case highlights the importance of including this in the differential diagnosis of acute vision loss.
\end{abstract}

Key words: branch retinal artery occlusion (BRAO), pediatric population, oral contraception

Received: December 12, 2014; Accepted with revision: June 3, 2015; Available online: July 3, 2015

http://dx.doi.org/10.5507/bp.2015.031

Department of Ophthalmology, Faculty of Medicine in Hradec Kralove, Charles University in Prague and University Hospital Hradec Kralove, Hradec Kralove

Corresponding author: Alexandr Stepanov, e-mail: stepanov.doctor@gmail.com

\section{INTRODUCTION}

Branch retinal artery occlusion (BRAO) causes obstruction of blood flow in the distribution of the affected vessel, leading to ischemia and reorganization of the retinal layers.

Artery occlusion occurs primarily in the elderly, with clinical findings suggestive of atheromatous emboli ${ }^{1}$. It is an extremely rare diagnosis in the pediatric population and the etiology with risk factors of retinal artery occlusion are poorly understood in younger individuals ${ }^{2}$. We report a rare case of a girl with the transient branch retinal artery occlusion.

\section{CASE REPORT}

A 15-year-old Caucasian girl reported to the eye clinic with the complaint of sudden loss of the inferior half of her visual field and painless decreased vision in her right eye. She denied floaters, flashes or other associated symptoms. She had no history of infectious disease, trauma, systemic malignancy, or other systemic complaint. A detailed history for suggestive cause of occlusion revealed that she had been on oral contraceptives (Logesta) at the time of occurrence right eye problems. Best corrected visual acuity was $20 / 100$ in the right eye and $20 / 20$ in the left eye. Intraocular pressure was $20 \mathrm{~mm} \mathrm{Hg}$ in both eyes measured by Goldmann applanation tonometry. Pupils were round and reactive in both eyes without evi- dence of relative afferent pupillary defect in either eye. Biomicroscopy of the anterior segment was unremarkable. Dilated fundus examination revealed normal findings in the left eye; in the right eye there was intensive edema of the superior half of the retina, but the vessels were absolutely normal (Fig. 1). No embolus was noted. Confrontation fields (standard automated perimetry, program test 30-2) showed a visual field defect corresponding with affected area in the right eye and normal visual field in the left eye. Fundus fluorescein angiography (FAG) and indocyanine green angiography (ICG) showed normal arteriovenous transit time in both eyes throughout the examination (Fig. 2). Optical coherence tomography (OCT) showed thickening associated with hyper-reflectivity of the inner retina with shadowing of the photoreceptors and retinal pigment epithelial layer (Fig. 3 ). Buried drusen of the optic nerve cup were excluded by ultrasonography examination. The girl was admitted to the pediatric hospital for further evaluation. Magnetic resonance imaging of the brain, electrocardiogram, echocardiography, chest radiographs, visual evoked potential and ultrasound examination of the abdomen were normal. Evaluation for potential stroke factors revealed no suggestive family history. Blood investigations including complete blood count with erythrocyte sedimentation rate, platelet count, serum lipids, prothrombin/activated partial thromboplastin time, autoimmune markers and serum homocysteine were all within normal limits. Screening for congenital metabolic diseases was negative. No ocular therapy was offered, and the patient was advised to 


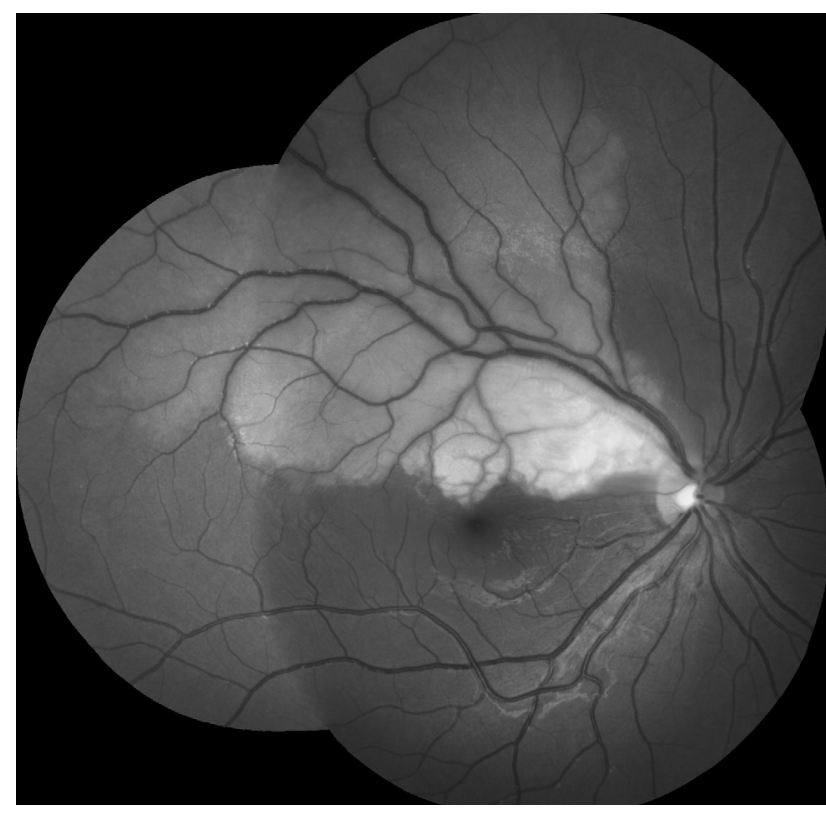

Fig. 1. Right eye. Intensive edema of the superior half of the retina, but the vessels are absolutely normal.

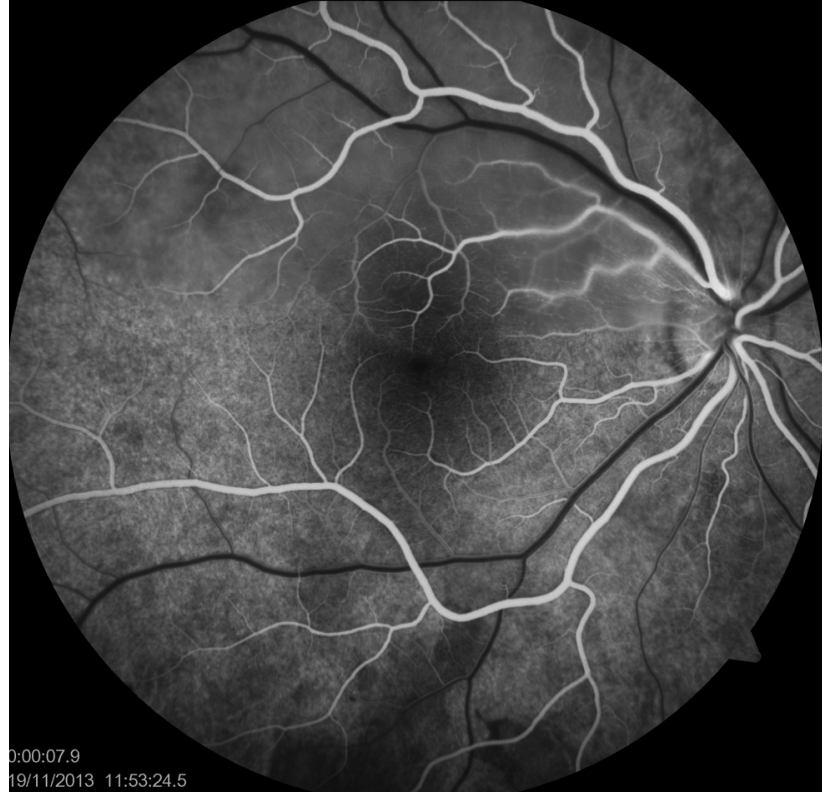

Fig. 2. FAG. Normal retinal vasculature on the right eye due retinal arterial stage.
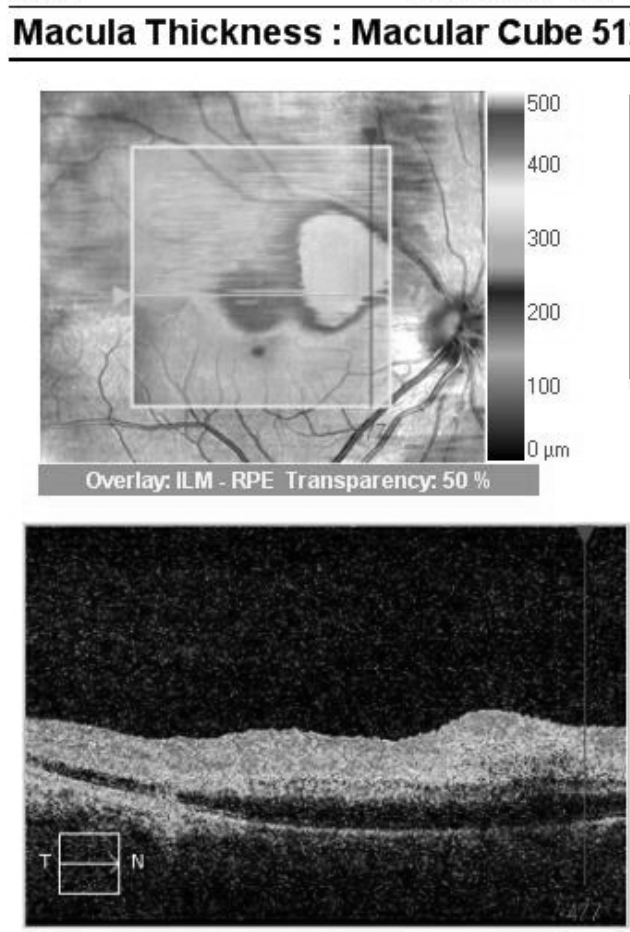

\section{OD O O OS}

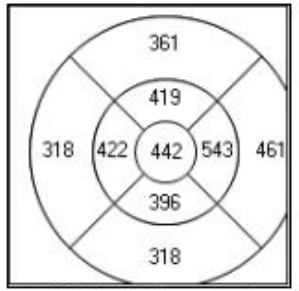

ILM-RPE Thickness ( $\mu \mathrm{m})$
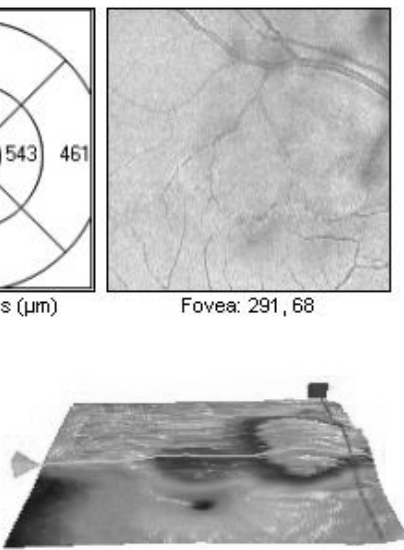

ILM - RPE

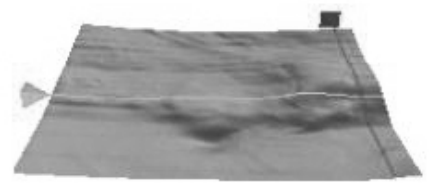

Fig. 3. Right eye. OCT shows thickening associated with hyper-reflectivity of the inner retina with shadowing of the photoreceptor and retinal pigment epithelial layer.

return for regular follow-up examination. At the first evaluation 1 month after presentation, the retinal edema had resolved remarkably, and visual acuity of the right eye had improved to a value of 20/20; however, the superior half of the retina was atrophic. The condition is evaluated as transient branch retinal artery occlusion by the course of the disease. At 3 months, the retinal edema had completely resolved ophthalmoscopically, and changes were observed in the superior half of the foveal reflex. Confrontation fields showed improvement in the prime inferior visual field defect of the right eye. Optical coherence tomography line scan showed no hyper-reflectivity, but the inner retina was attenuated. 


\section{DISCUSSION}

Retinal artery occlusion is an extremely rare diagnosis in the pediatric population. The incidence of retinal arterial obstruction in patients under the age of 30 years has been estimated at less than 1 in 50,000 outpatients ${ }^{2}$. Multiple mechanisms exist that cause arterial occlusion in the retina. Frequent etiologies include hypercoagulable states, hyperhomocysteinemia, vasculitis, emboli from cardiac valvular disease, and other risk factors such as smoking, use of oral contraceptives, and vasospasm such as in a history of migraine ${ }^{2-7}$.

There are few published reports of retinal artery occlusion associated with hyperhomocysteinemia in children ${ }^{7-9}$. Abnormal accumulation of homocysteine in plasma, as found in this inborn error of metabolism of the amino acid methionine, also has a toxic effect on endothelial cells resulting in arteriosclerosis, and arterial and venous thromboembolic events at a young age. Elevations in the plasma homocysteine concentration can occur because of genetic defects in the enzymes involved in homocysteine metabolism, nutritional deficiencies in vitamin cofactors (folate, vitamin B6, or vitamin B12), or other factors including some chronic medical conditions and drugs. Ratra and Dhupper suggested that the common systemic association of retinal artery occlusion in the Indian population was hyperhomocysteinemia ${ }^{8}$. Karth et al. described a previously healthy 7-week-old boy who developed bilateral central retinal artery occlusion in the presence of hyperhomocysteinemia ${ }^{9}$.

Talmon et al. documents retinal arterial occlusion in a child caused by a combination of factor $\mathrm{V}$ Leiden and thermolabile methylene tetrahydrofolate reductase homozygosity ${ }^{10}$. Although factor V Leiden has been associated clearly with venous thrombosis, most studies have failed to demonstrate a clear association between isolated factor V Leiden and arterial thrombosis. In this disorder, the Leiden variant of factor $\mathrm{V}$ cannot be inactivated by the anticoagulant protein activated protein $\mathrm{C}$, so clotting is encouraged ${ }^{11}$.

Saliba et al. described bilateral branch retinal artery occlusions in a 14-year-old girl with Susac syndrome ${ }^{3}$. This is a rare disorder with a clinical triad of encephalopathy, branch retinal artery occlusions, and hearing loss. It is caused by a microangiopathy affecting the precapillary arterioles of the brain, retina, and inner ear.

In this instance, systemic studies including hypercoagulation, autoimmune and infectious workups, hyperhomocysteinemia were negative, but patient use of oral contraceptives.

The incidence of ocular complication from birth control pills is estimated to be 1 in 30,000 (ref. ${ }^{12}$ ). Numerous case reports were published concerning the occurrence of a variety of eye disorders in women using oral contraceptives $^{13,14} .2$ cases of young woman who had taken birth control pills and in whom arterial vascular occlusion of retina developed was reported by Leff et al. ${ }^{12}$. Giromi et al. published a case report a central retinal artery occlusion in a young woman after ten days of drospirenone contain- ing oral contraceptive ${ }^{15}$. Several mechanisms have been proposed to explain the increased stroke risk associated with oral contraceptives, particularly induction of a hypercoagulable state and raised blood pressure. Harris and colleagues found that levels and activity of tissue factor pathway inhibitor were decreased by $25 \%$ and $29 \%$, respectively, among women on oral contraceptives ${ }^{16}$. Not only is there decreased inhibition of the extrinsic pathway, but there also is evidence for activation of this pathway with increases in plasma factor VIIa activity and prothrombin fragments 1 and 2. Other studies have similar results ${ }^{17}$. Increased levels of procoagulants (Factor I, VII, VIII, IX, $\mathrm{X}, \mathrm{XII}$, and XIII), decreased anticoagulant factors protein $\mathrm{S}$ and antithrombin, and acquired activated protein $\mathrm{C}$ resistance have all been described ${ }^{18}$. Furthermore, changes in lipid profile show increase in LDL cholesterol and decreases in HDL cholesterol fractions among women on oral contraceptives ${ }^{19}$.

In our case the condition is evaluated as transient branch retinal artery vasospasm due use of oral contraceptives.

\section{CONCLUSIONS}

This is a rare report of branch retinal artery occlusion in a girl. Though retinal arterial obstruction is rare in the pediatric population, our case highlights the importance of including this in the differential diagnosis of acute vision loss. This case is unique with regard to the age of the patient and the presentation of transient vasospasm.

\section{ACKNOWLEDGEMENT}

Author contributions: All co-authors have read the final manuscript within their respective areas of expertise and participated sufficiently in the study to take responsibility for it and accept its conclusions.

Conflict of interest statement: The authors state that there are no conflicts of interest regarding the publication of this article.

\section{REFERENCES}

1. Kollarits CR, Lubow M, Hissong SL. Retinal strokes: I: Incidence of carotid atheromata. JAMA 1972;222:1273-5.

2. Brown GC, Magargal LE, Shields JA, Goldberg RE, Walsh PN. Retinal arterial obstruction in children and young adults. Ophthalmology 1981;88:18-25.

3. Saliba M, Pelosse B, Momtchilova M, Laroche L. Susac syndrome and ocular manifestation in a 14-year-old-girl. J Fr Ophthalmol 2007;30:1017-22.

4. Ann M, Cho NC. Central retinal artery occlusion in herpes zoster ophthalmicus. J Pediatr Ophthalmol 2002;39:123-4.

5. Lagunju A, Brown BJ. Adverse neurological outcomes in Nigerian children with sickle cell disease. Int J Hematol 2012;96:710-8.

6. Greven CM, Slusher MM, Weaver RG. Retinal arterial occlusions in young adults. Am J Ophthalmol 1995;120:776-83.

7. Coban-Karatas M, Erol I, Ozkale Y, Yazici N. Central Retina Artery Occlusion in a 13-Year-Old Child as a Presenting Sign of 
Hyperhomocysteinemia Together With High Lipoprotein(a) Level. Pediatric Neurology 2013;49:138-40.

8. Ratra D, Dhupper M. Retinal artery occlusion in the young: Systemic associations in Indian population. Indian J Ophthalmol 2012;60(2):95-100.

9. Karth P, Singh R, Kim J, Costakos D. Bilateral central retinal artery occlusions in an infant with hyperhomocysteinemia. J AAPOS 2012;16(4):398-400.

10. Talmon T, Scharf J, Mayer E, Lanir N, Miller B, Brenner B. Retinal arterial occlusion in a child with factor $\mathrm{V}$ Leiden and thermolabile methylene tetrahydrofolate reductase mutations. Am J Ophthalmol 1997;124(5):689-91.

11. Press RD, Bauer KA, Kujovich JL, Heit JA. Clinical utility of factor V leiden (R506Q) testing for the diagnosis and management of thromboembolic disorders. Arch Pathol Lab Med 2002;126(11):1304-18.

12. Leff S P. Side effect of oral contraceptives: occlusion of branch artery of retina. Bull Sinai Hosp Detroit 1976;24(4):227-9.

13. Wood JR. Ocular complications of oral contraceptives. Ophthalmic Sem 1977;2:371-402.
14. Vessey MP, Hannaford P, Mant J, Painter R, Frith P, Chappel D. Oral contraception and eye disease: findings in two large cohort studies. Br J Ophthalmol 1998;82(5):538-42

15. Girolami A, Vettore S, Tezza F, Girolami B. Retinal central artery occlusion in a young woman after ten days of a drospirenone-containing oral contraceptive. Thromb Haemost 2007;98(2):473-4.

16. Harris GM, Stendt CL, Vollenhoven BJ, Gan TE, Tipping PG. Decreased plasma tissue factor pathway inhibitor in women taking combined oral contraceptives. Am J Hematol 1999;60:175-80.

17. Dahm A, Van Hylckama Vlieg A, Bendz B, Rosendaal F, Bertina RM, Sandset PM. Low levels of tissue factor pathway inhibitor (TFPI) increase the risk of venous thrombosis. Blood 2003;101(11):4387-92.

18. Alhenc-Gelas M, Plu-Bureau G, Guillonneau S, Kirzin JM, Aiach M, Ochat N, Scarabin PY. Impact of progestagens on activated protein C (APC) resistance among users of oral contraceptives. J Thromb Haemost 2004;2(9):1594-600.

19. Knopp RH, Broyles FE, Cheung M, Moore K, Marcovina S, Chandler WL. Comparison of the lipoprotein, carbohydrate, and hemostatic effects of phasic oral contraceptives containing desogestrel or levonorgestrel. Contraception 2001;63(1):1-11. 